Session 1348

\title{
Development of Multimedia Instructional Tools for Strength of Materials
}

\author{
Jack Zecher, Justin Davis, Heather Deaton and Deric Pawlaczyk \\ Mechanical Engineering Technology Department \\ Indiana University-Purdue University Indianapolis
}

\begin{abstract}
This paper describes a series of multimedia based instructional lessons that are being developed to enhance a Strength of Materials course. Use of these lessons will help students to more rapidly understand many concepts that are difficult to describe on the printed pages of a textbook or in the traditional classroom chalkboard lecture. These computer based lessons consist of realistic images, animations of parts deforming as loads are applied, color stress contours, and simulated "pencil-on-paper solutions." These lessons provide an alternative learning environment which can be used as a supplement to the traditional classroom. The process of how a combination of three-dimensional images, animation sequences, and audio narrations were used to create these lessons is discussed. This includes the various software packages were used and how these various multimedia components were combined and delivered.
\end{abstract}

Introduction

There are currently many areas in which computer based products are being used to improve instruction in Strength of Materials courses for engineering technology students. Several instances ${ }^{1,2}$ involve the use of advanced analytical software tools, such as finite element analysis to allow students to perform calculations more sophisticated than those normally covered in the course. These types of computer applications provide students with the means to explore problems beyond the scope of the typical textbook; however, these tools require a large amount of time and effort in order to provide a minimal amount of beneficial instructional content.

With the advent of online courses, computers connected to the internet have been used to facilitate the distribution of course materials. Many courses are now taught where course notes and solutions to homework problems and previous exams are posted for students on the web. By eliminating barriers of time and distance, this improved distribution mechanism makes it easier to provide reference materials and examples to students. Much of the material delivered in this manner, however, is no different from that which could be photocopied and handed out in the classroom.

The project described in this paper takes advantage of the multimedia capabilities of the computer to improve the quality of material used to describe example problems. A combination 
of three-dimensional modeling and rendering programs are used to produce images of the parts involved in the example problems so that students gain a better understanding of the object being analyzed. Animation sequences are used to demonstrate how parts deform under the applied load(s). Displays are produced that illustrate how the resulting stresses are distributed on crosssections, or over the surface of the part. A simulated "pencil-on-paper solutions" portion helps to demonstrate and reinforce the desired problem-solving methodology skills that students should develop in the course. Each of the example problems includes a voice-over audio narration to provide another sensory means of explaining the problem and the steps necessary in its solution.

The Course

The course which will use the multimedia material is the Applied Strength of Materials (MET 211) course at IUPUI. It is a first semester sophomore course that has the prerequisite of Applied Statics (MET 111) and a co-requisite of Calculus for Technology I (MATH 221). The topics covered in this course include the following:

.Tensile \& Compressive Stresses

.Shear Stresses

.Deformation \& Strain

.Thermal Effects

.Mechanical Properties of Materials

.Stress Concentrations

.Torsional Stresses and Deformations

.Shear Forces \& Bending Moments in Beams

.Stresses in Beams

.Deflection of Beams

.Combined Stresses

.Columns.

Ideally, lessons similar to the one described in this paper will eventually be developed to support all of the different topics included in the course. It was decided to start the development process with an example problem that illustrated the subject of Combined Stresses. This topic is one that is often misunderstood by the students because two normal stresses are superimposed. The goal was to provide the student a multimedia lesson that explained how axial stress and bending stress are combined. This is usually expressed mathematically as:

$$
\sigma= \pm \frac{P}{A} \pm \frac{M c}{I}
$$

\section{Project Overview}

The project team consisted of Professor Zecher, who teaches the Applied Strength of Materials course at least once each year, and the three co-authors of this paper, who were senior Computer Graphics Technology students. The students worked on the project as part of their CGT 411 Contemporary Problems in Computer Graphics course. Professor Zecher served as the students' client and technical consultant - providing the students with an outlined of the desired product. 
The students then were responsible for creating the graphical images and combining them into a multimedia presentation.

Prior to starting the project, two existing multimedia products were studied and evaluated. The first was the MecMovies (C) series of mechanics of materials lessons that were developed at the University of Missouri by Philpot, T., et $\mathrm{al}^{3}$. The second was a series of web-based "chalkboard" example problems: "e-courses" from the University of Oklahoma that were developed by Gramoll, K. . . It was determined that the strongest aspect of the MecMovies (C product was its excellent graphics, while the e-course product was attractive because it contained an audio script which explained each step of the problem solution. It therefore became a goal, to incorporate both of these desirable aspects into the product to be developed.

The project began with a "storyboard" outline of the images to be presented to the students. Along with the sketches of the images, a script was written based on the actual step-by-step process of solving the problem. The narration of the script was captured electronically, and saved as .wav files. The Sound Forge program was used during the recording process and the resulting file was saved as a $44 \mathrm{kHz}, 16 \mathrm{bit}-\mathrm{mono}$ sound file. This sound file was then edited to break the file into a number of small files which allowed mistakes to be deleted and to easily move the "sound bites" back and forth along a time line in order to correctly match the corresponding image being displayed.

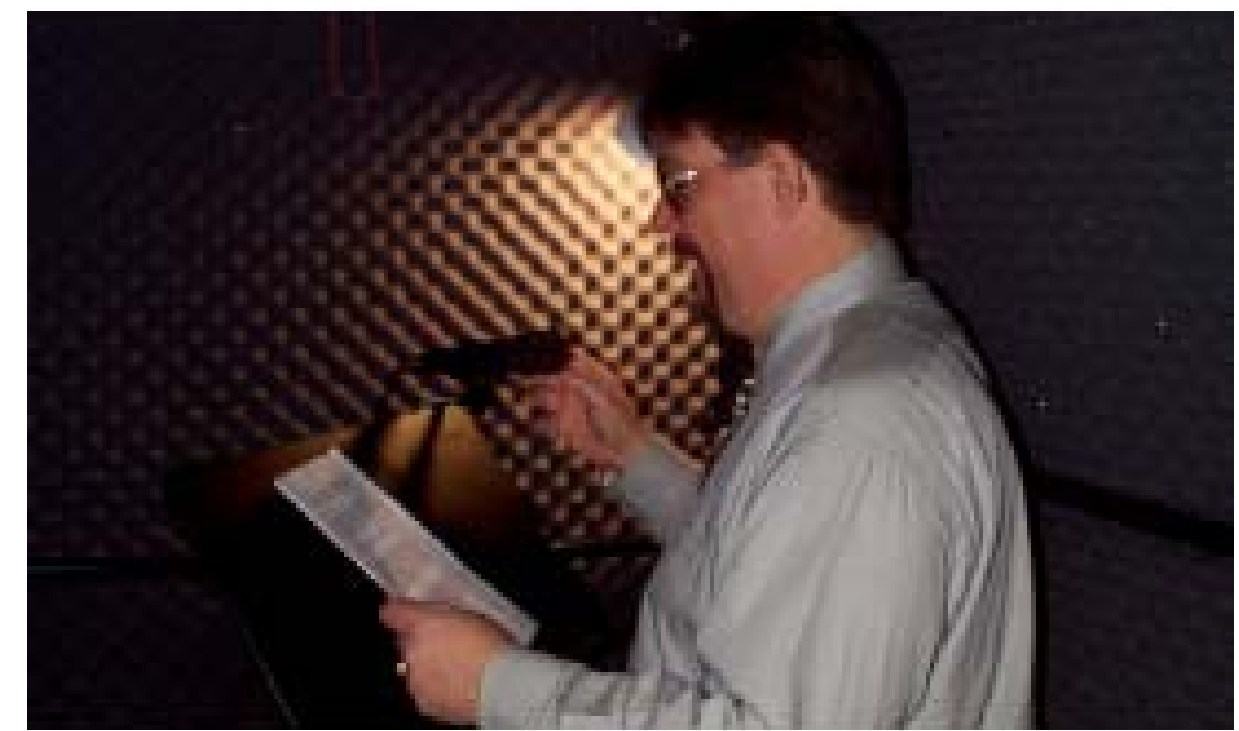

Figure 1 Recording of the "voice-over" being recorded in a sound booth

Graphical images similar to that shown in figure 2 were created with a number of different software packages: ProEngineer, Macromedia Freehand, etc.. 


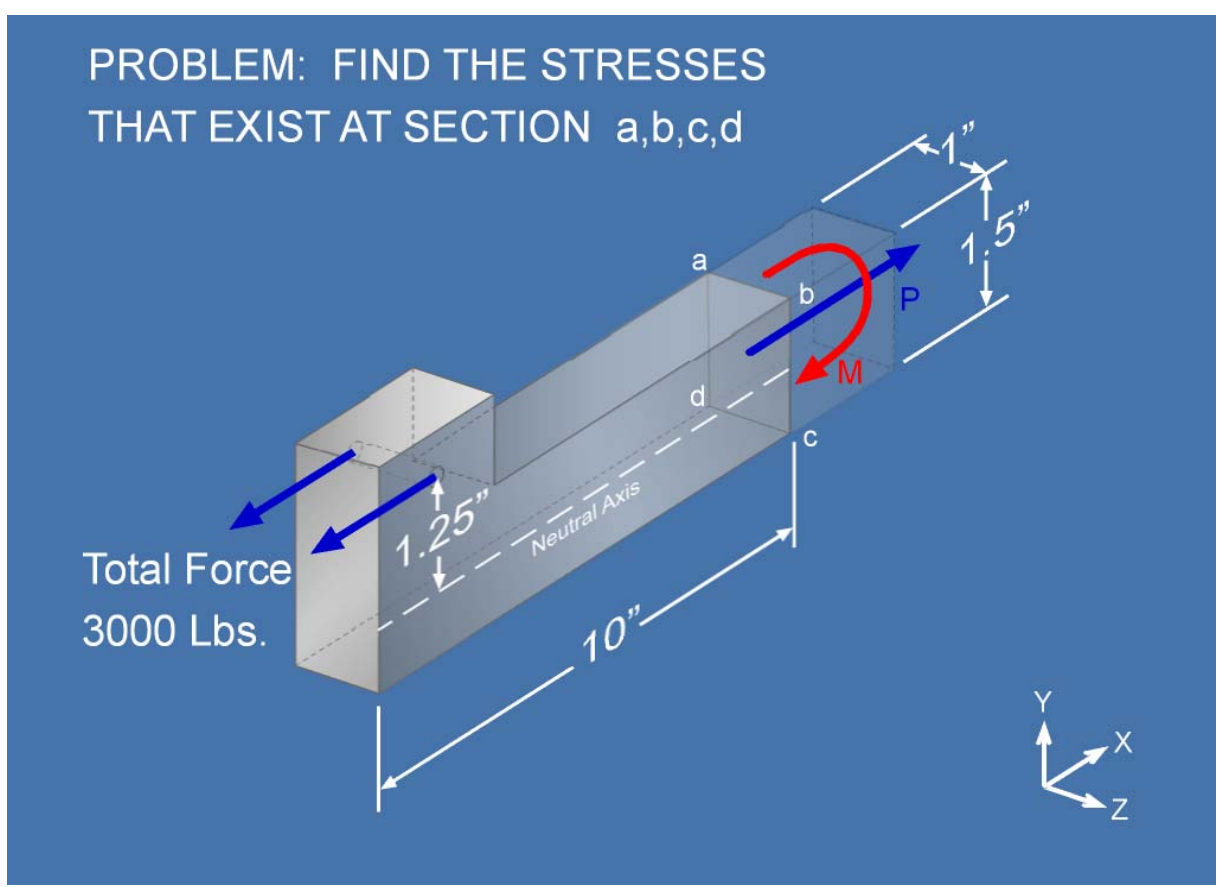

Figure 2 First image displayed which presents the problem to be solved

Because students in the Applied Strength of Materials course are required to turn their homework problems in on graph paper, it was decided to also present the numerical solution on simulated graph paper. This was done by first scanning a white sheet of paper that contained the completed problem. Then using Adobe PhotoShop ${ }^{\mathrm{TM}}$ the image was enhanced to contain a background that was the color of graph paper, along with the grid lines. Also, a series of images were produced, each revealing a little more of the solution - so that as the narration of the problem progressed, the corresponding image would be revealed.
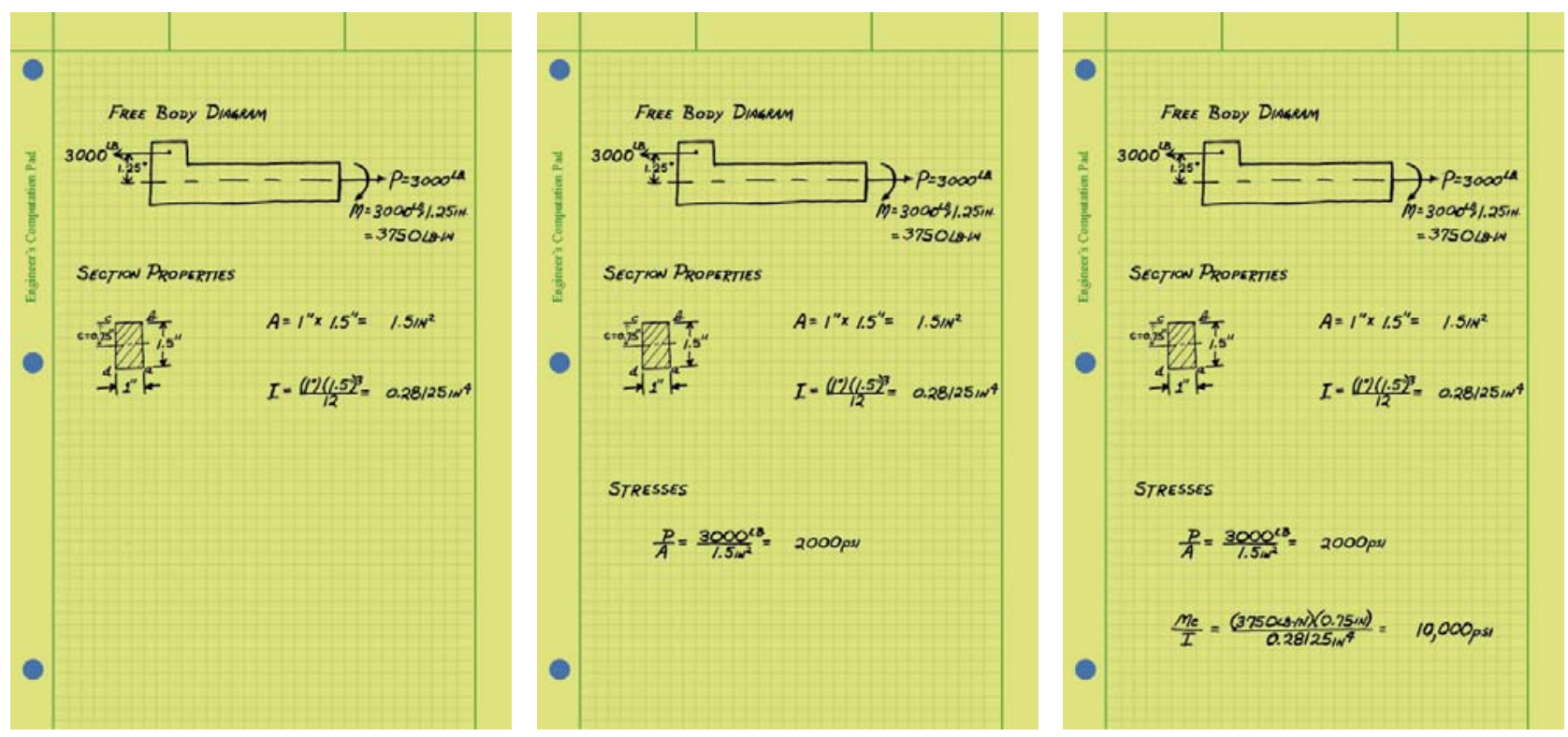

Figure 3 Three of the images of the problem solution, which when displayed sequentially gives the appearance of the solution being written 
In order to produce a "video" of the sequence of images and the audio narration, the Adobe Premiere ${ }^{\mathrm{TM}}$ program was used. The Premiere ${ }^{\mathrm{TM}}$ program provides a graphical interface in its Construction window that allows the graphical images to be easily synchronized with the audio narration. Each individual bitmap file can easily be stretched or compressed along a timeline, thereby giving the user control as to the length of time each individual image would appear on the screen. The corresponding audio "sound bites" are then placed in a parallel track as shown in figure 4. Adobe Premiere ${ }^{\mathrm{TM}}$ also has the capabilities to build transitions between images, which provided the appearance of images fading in or out. The resulting "video" was saved as an .avi file.

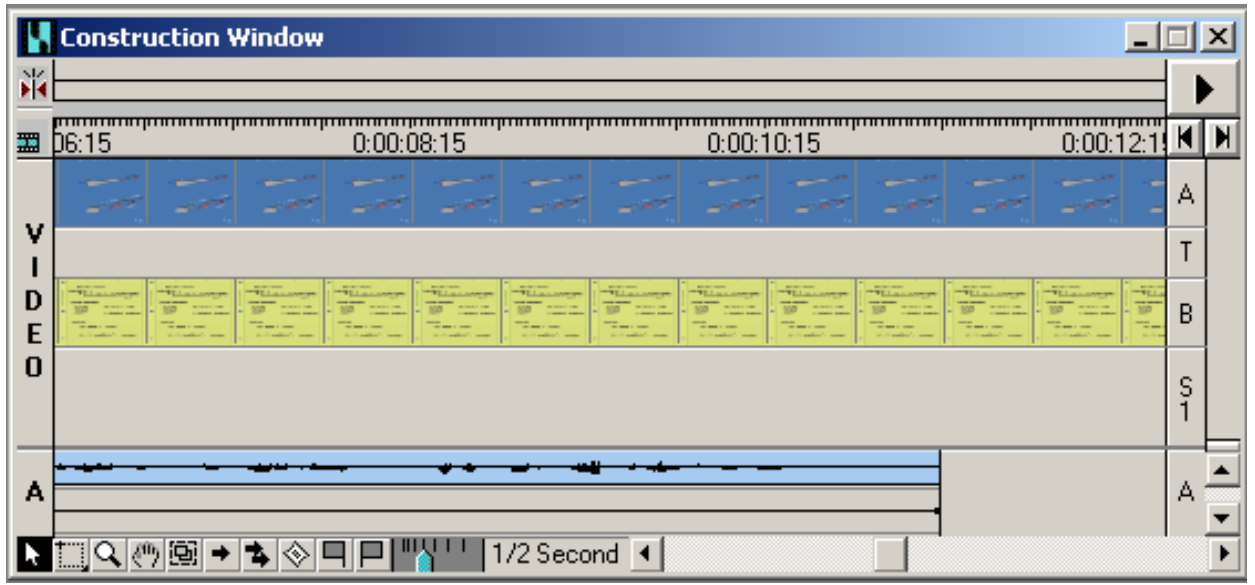

Figure 4. Construction window of Adobe Premiere ${ }^{\mathrm{TM}}$

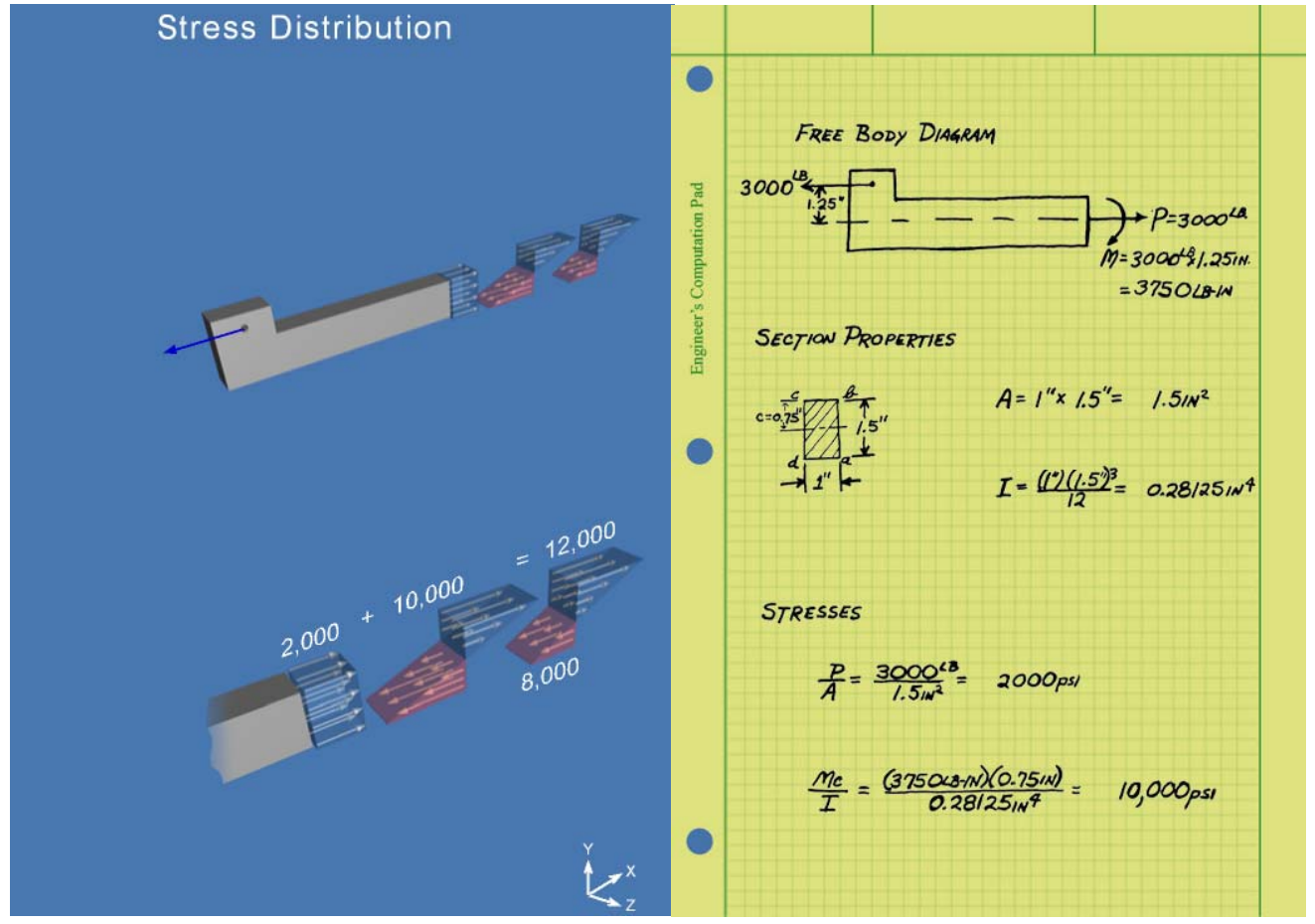

Figure 5 Example display presented on the screen, that contains both graphical image and numerical solution on graph paper 
In addition to the superimposed stress distributions on the cross section as shown in figure 5, other types of graphical displays and animations are provided. For example, figure 6 illustrates the display of stress contours over the entire surface of the part. These additional types of images are used to convey a better understanding of how the stresses are distributed throughout the entire part.

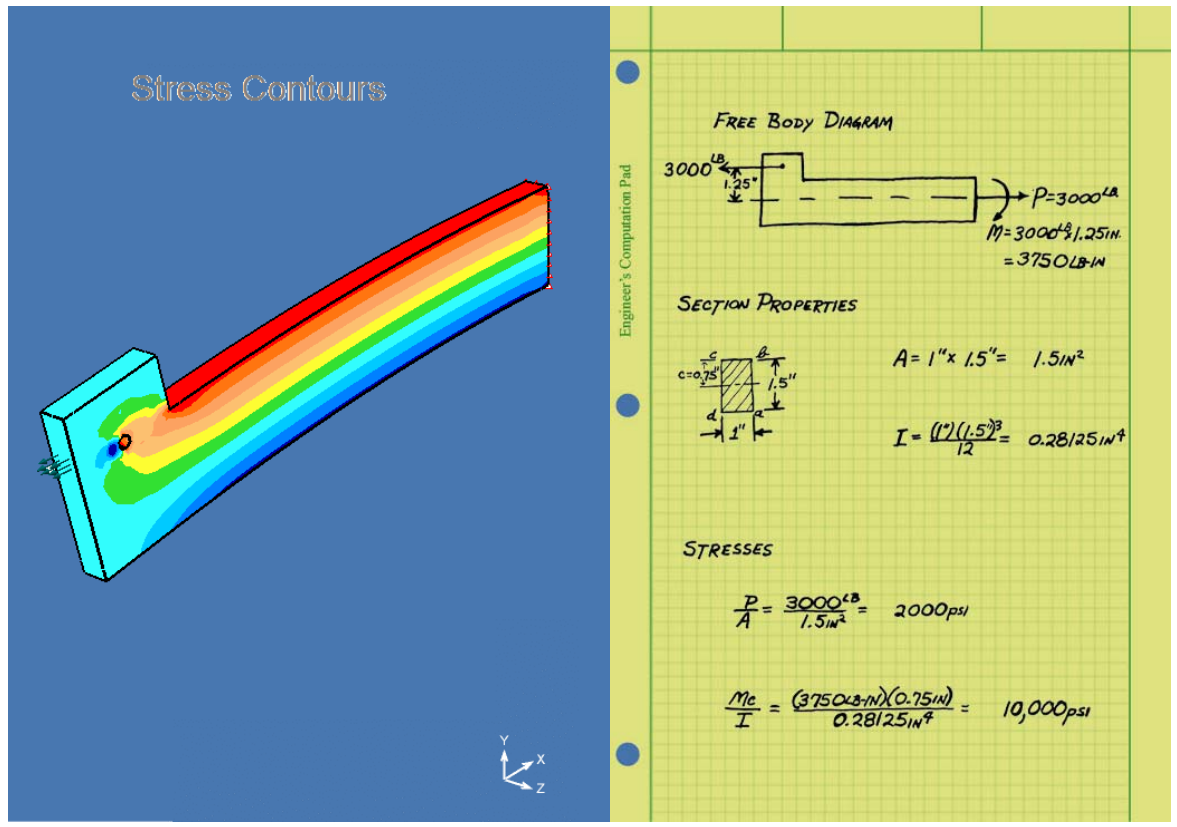

Figure 6 Example display that contains both an image of the stress contours and the numerical solution on graph paper

Once the.$a v i$ files were created, Macromedia Director ${ }^{\mathrm{TM}}$ was used to control which lesson's .avi file should be played as well to provide the user with a VCR-like interface to play the .avi files as shown in figure 7 . Included in the VCR-like interface are play, pause and reverse buttons along with a slider control that allow the animation and audio narration to be repositioned, replayed or paused. This allows the student to review any portion of the video as many times as desired.

\section{Stop/Play $\rightarrow>$ D回 四 I}

\section{Figure 7 VCR-like interface is used to play the resulting video files}

\section{Conclusions}

The realistic three-dimensional displays, animations of the parts deforming as loads are applied, and resulting stress contour displays allow students to gain more insight than would be possibly from a static picture in a textbook or drawing on the blackboard. These improved images and animations help to accelerate the mental visualization process that is required for comprehending and solving these types of problems. Because the development of these lessons did not begin until the fall 2004 semester only one group of students have had an opportunity to use two of the 
multimedia based lessons. Formal assessment of these lessons' impact has not yet been done. However, students' comments so far have all been positive, with many requests for additional multimedia lessons to be developed.

Currently only three lessons, similar to the one discussed in this paper have been completed. The development of additional lessons is an ongoing process. However, because of the labor intensive nature of developing multimedia material it is hoped that external funding will be obtained to help support the additional work required to complete this project.

The use of these lessons should help to support a web-based version of the Strength of Material course. The multimedia material should help to "close the gap" that often exists in many web based courses that are actually an on-line version of independent study.

\section{References}

1 Earley, Ronald, "Use of FEA in an Introductory Strength of Materials Course", American Society for Engineering Education Annual Conference 1998

2. Logue, L. J., Hall, K. A., "Introducing Finite Element Analysis in an MET Strength of Materials Course, American Society for Engineering Education Annual Conference 2001

3 http://web.umr.edu/ mecmovie/index.html

4 https://ecourses.ou.edu/cgi-bin/navigation.cgi?course $=$ me

Jack Zecher, P.E.

Professor of MET at IUPUI has been with the University since 1983. Jack teaches courses in the mechanical design field as well as Computer Aided Design related subjects. He has authored two books: Computer Graphics for CAD/CAM Systems, published by Marcel Dekker in 1994, and Finite Element Analysis Tutorial Using Algor version 14, published by Schroff Development Corporation in 2003. He has also authored multimedia CDs that are packaged with several different CAD tutorials published by Schroff Development Corporation. He holds a Master of Science degree in Mechanical Engineering Technology from Western Michigan University and is a registered Professional Engineer in the state of Indiana.

Justin Davis

Recently graduated (December 2004) with his Bachelor of Science degree in Computer Graphics Technology from IUPUI

Heather Deaton

Recently graduated (December 2004) with her Bachelor of Science degree in Computer Graphics Technology from IUPUI

Deric Pawlaczyk

Recently graduated (December 2004) with his Bachelor of Science degree in Computer Graphics Technology from IUPUI 